\title{
Composición Corporal y Somatotipo de Futbolistas Chilenos Juveniles Sub 16 y
}

\author{
Body Composition and Somatotype of Chilean Soccer Players Sub 16 y Sub 17
}

"Carlos Jorquera Aguilera; **Fernando Rodríguez Rodríguez; "María Ignacia Torrealba Vieira \& ***Fernando Barraza Gómez

JORQUERA, A. C.; RODRÍGUEZ, R. F.; TORREALBA, V. M. I. \& BARRAZA, G. F. Composición corporal y somatotipo de futbolistas chilenos juveniles sub 16 y sub 17. Int. J. Morphol., 30(1):247-252, 2012.

RESUMEN: El fútbol juvenil, se profesionaliza cada día más, al punto de incluir a los jóvenes en los equipos adultos que juegan en los Torneos Profesionales. Para que esto ocurra es necesario que los jóvenes deportistas estén físicamente aptos para enfrentar las exigencias físicas del futbol profesional. Se evaluó antropométricamente a un total de 217 sujetos distribuidos en dos grupos, sub 16 y sub 17. Los jóvenes futbolistas, presentan diferencias en la estructura corporal con respecto a futbolistas profesionales, demostrando una falta de desarrollo madurativo y deportivo, que según la evidencia, los hace menos aptos físicamente, para enfrentar exigencias físicas, típicas del futbol profesional. La masa muscular de los sujetos es menor que la de futbolistas profesionales, por lo que no están aptos para ser incluidos dentro del equipo de honor en los partidos de competencia, con el riesgo de sufrir lesiones y de tener un rendimiento físico menor.

PALABRAS CLAVE: Antropometría; Composición corporal; Masa muscular; Fútbol.

\section{INTRODUCCIÓN}

Las características antropométricas de los sujetos, pocas veces son consideradas en la selección de los jóvenes deportistas, para ciertos niveles o para categorizarlos en distintas disciplinas deportivas, sino que muchas veces se pone la atención en las habilidades que cada individuo tiene en su respectiva especialidad, sin considerar que una vez que los jóvenes pasan a categorías adultas, la masa muscular y la fuerza, son elementos que priman en los deportes como el futbol, por lo que un sujeto con mejores características físicas, como mayor estatura, mayor masa muscular o menos grasa, es capaz de correr más km por partido, saltar más alto, y ser más eficiente que un rival que sea delgado y que tenga mejor habilidad, facilitando el rendimiento intermitente de alta intensidad por periodos largos de tiempo, que es característico del futbol competitivo (Krustrup \& Bangsbo, 2001; Krustrup et al., 2003, 2005; Mohr et al., 2003, 2005).

En un estudio hecho en jugadores portugueses de futbol de entre 13 y 15 de edad, se determinó que el entrenamiento físico favorece el desarrollo de la capacidad aeróbica, pero que otras características físicas, no entrenables, como la estatura y peso, influyen positivamente sobre el salto vertical y un sprint de 30m, respectivamente (Malina et al., 2004).
Por otro lado, los deportistas jóvenes, ven mejorado su rendimiento físico a medida que su maduración se completa (Malina et al.). Por lo tanto aquellos jugadores elegidos para el deporte, según sus características técnicas, sin considerar su estructura física, pueden fracasar profesionalmente al no tener la estatura adecuada, la masa muscular idónea, o lesionarse crónicamente debido a su falta de fortaleza física (Arnason et al., 2004), la cual es característica del futbol europeo, donde deben optar nuestros jugadores para desarrollarse deportivamente.

En este estudio demostraremos y compararemos las características antropométricas adecuadas para el futbol profesional entre jugadores jóvenes y adultos.

\section{MATERIAL Y MÉTODO}

Los sujetos del estudio son jóvenes futbolistas de sexo masculino, de las categorías sub 16 y sub 17, pertenecientes a equipos profesionales de futbol, donde sus planteles de honor, participan en la Primera División A del futbol chile-

\footnotetext{
Escuela de Nutrición y Dietética, Facultad de Medicina, Universidad Mayor, Chile.

** Laboratorio de Motricidad Humana, Escuela de Educación Física, Laboratorio de Antropología Física y Anatomía Humana, Instituto de Biología, Pontificia Universidad Católica de Valparaíso, Chile.

**** Laboratorio de Motricidad Humana, Escuela de Educación Física, Pontificia Universidad Católica de Valparaíso, Chile.
} 
no. Todos ellos accedieron al estudio de forma voluntaria y bajo un consentimiento informado.

Se evaluó a un total de 216 sujetos distribuidos en dos grupos, sub 16 y sub 17. Las evaluaciones se realizaron entre los años 2010 y 2011. Estos sujetos jóvenes pertenecían a 6 equipos de futbol, Colo-Colo $(\mathrm{n}=46)$, Universidad de Chile ( $n=51)$, Palestino $(n=26)$, Unión Española $(n=16)$, O’higgins de Rancagua ( $\mathrm{n}=50)$ y Everton $(\mathrm{n}=28)$. Los datos de medidas básicas se observan en la Tabla I.

La evaluación antropométrica se realizó en las primeras horas de la mañana, con la menor ropa posible y después del vaciado urinario, en los mismos lugares de entrenamiento de cada equipo. Se midió bajo el protocolo de marcaje de la International Society for the Advancement of Kineatropometry (ISAK) y de evaluación, descritos por Norton \& Olds (1996) y Drinkwater (1984), para el procedimiento de medición completa de variables.

Para la determinación de la composición corporal, se consideró el tejido adiposo, muscular, óseo, residual y de piel, utilizando las ecuaciones propuestas por Kerr (1988). Además se estima la masa muscular por segmentos de los miembros superiores e inferiores, con la propuesta hecha por Rodríguez et al. (2010). Se determinó la forma corporal de los sujetos a través del método del somatotipo de Carter (Carter \& Heath, 1990; Carter, 2002).

Para la aplicación del método antropométrico se utilizó el Kit Gaucho Pro "Mercosur", fabricado en Argentina bajo licencia de Rosscraft Canadá para su comercialización en el Continente Americano (excepto EE.UU, Canadá y México).
El kit antropométrico está compuesto por los instrumentos: Campbell 20 (antropómetro largo), Campbell 10 (antropómetro corto), segmómetro, escuadra, calibrador de pliegues cutáneos o plicómetro, cinta métrica para perímetros, un estadiómetro para estatura y una balanza para el peso con precisión de 100 gramos.

La recolección de los datos se facilita por el asistente, quien ayuda al evaluador a registrar las variables, dicho asistente conocía las técnicas de medición lo que permitió un trabajo fluido y rápido, además aseguraba la secuencia correcta de los lugares de medición.

La ficha de recolección de los datos o proforma fue diseñada con la intención de mejorar la rapidez de la medición, con un orden que va desde superior a inferior de la posición anatómica. Se evaluaron 3 variables básicas, peso, estatura de pie. Y estatura sentado, 6 diámetros óseos, 10 perímetros y 6 pliegues cutáneos.

Se establecieron diferencias estadísticas a través de una prueba t entre las variables evaluadas y obtenidas como resultados, considerando un valor significativo de $\mathrm{p}<0,05$.

\section{RESULTADOS}

Los elementos importantes a considerar en el análisis de la composición corporal, pueden ser los valores procesados de datos, como por ejemplo la masa muscular o masa grasa estimada a través de formulas, así como también los datos sin someterlos a otras formulas, como por ejemplo la

Tabla I. Promedios y desviaciones estándar de la edad, peso, Talla e Índice de Masa Corporal (IMC) de los sujetos de evaluados de ambas categorías.

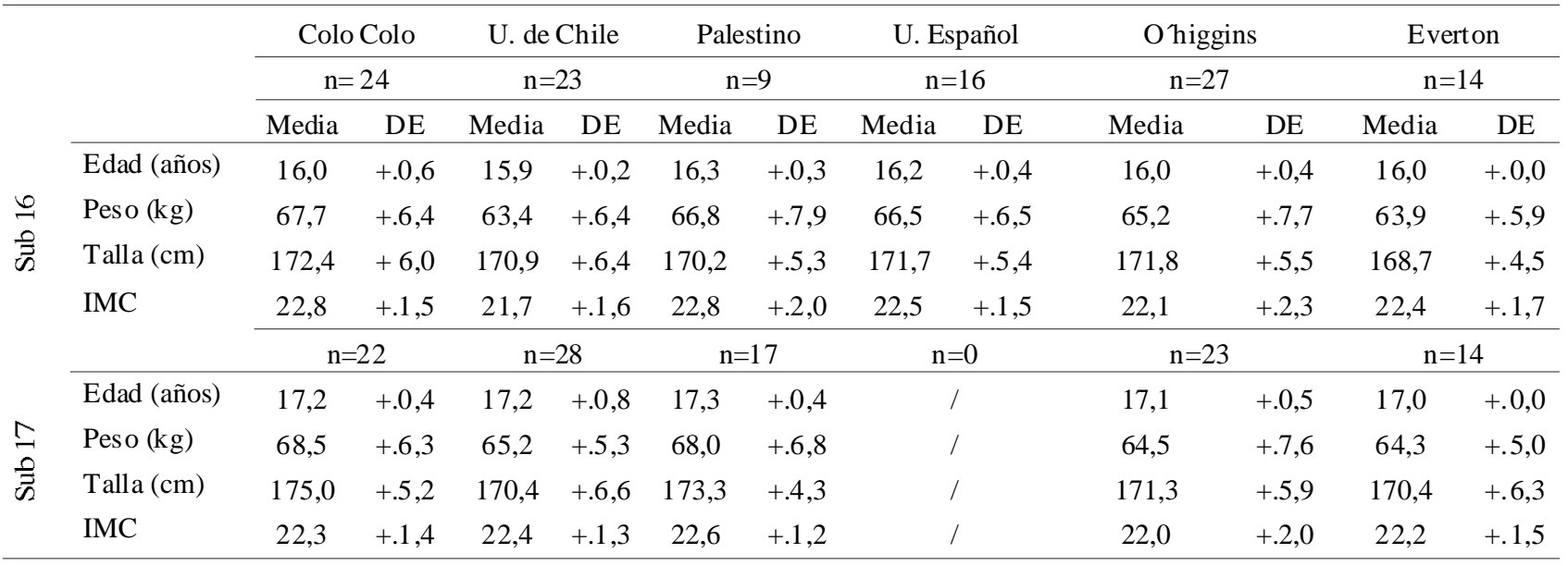

(*) Diferencias Significativas de la MMEI con una $\mathrm{p}<0,05$ en una prueba T, respecto a los demás equipos. (**)Diferencias Significativas de la $\sum 6$ Pliegues con una $\mathrm{p}<0,05$ en una prueba $\mathrm{T}$, respecto a los demás equipos. 
sumatoria de 6 pliegues en $\mathrm{mm}$, como un valor importante a destacar y que se presenta en la Tabla II. A través de esta sumatoria es posible tener una idea comparativa de la cantidad de grasa que trasciende cualquier método de estimación.

El equipo de O’Higgins, presenta una sumatoria de 6 pliegues, más baja que los otros equipos de las categorías sub 16 y sub 17.

Respecto de la masa grasa, no hay diferencias en la categoría sub 16 , pero si las hay en la categoría sub 17 , donde el equipo de O’Higgins, es el que presenta menor peso en grasa, siendo coherente con la menor sumatoria de 6 pliegues.

La masa muscular total de los sujetos de ambas categorías, no presentan diferencias estadísticas entre los grupos. Pero si las hay en la masa muscular de las miembros inferiores (MMEI) de la categoría sub 16, donde los equipos de U. de Chile, Palestino y U. Española, presentan un valor más bajo que el resto de los equipos. La masa muscular de los miembros inferiores de la categoría su 17, no presenta diferencias significativas.

Comparando entre las dos categorías, sub 16 y sub 17 , no se observan diferencias significativas en la masa grasa y masa muscular total.

La clasificación del Somatotipo, se realiza de acuerdo al los dos valores más altos presentados por el sujeto, es decir, si el valor más alto es el mesomorfismo, se utiliza la palabra completa para la descripción y si el segundo valor más alto es el ectomorfismo, se señala solo con las primeras cuatro letras, o sea, el sujeto es ecto-mesomorfico. De acuerdo a los anterior, los resultados del Somatotipo, en la cate-

Tabla II. Promedios y desviaciones estándar de la composición corporal según Kerr (1988), de la masa muscular por segmentos según Rodríguez (2010) y del Somatotitpo según Carter (2002).

\begin{tabular}{|c|c|c|c|c|c|c|c|c|c|c|c|c|c|}
\hline & & \multicolumn{2}{|c|}{ Colo-Colo } & \multicolumn{2}{|c|}{ U. de Chile } & \multicolumn{2}{|c|}{ Palestino } & \multicolumn{2}{|c|}{ U. Español } & \multicolumn{2}{|c|}{ O’Higgins } & \multicolumn{2}{|c|}{ Everton } \\
\hline & & Media & DE & Media & $\mathrm{DE}$ & Media & DE & Media & DE & Media & DE & Media & $\mathrm{DE}$ \\
\hline \multirow{13}{*}{$\begin{array}{l}\mathscr{0} \\
\stackrel{\theta}{\sigma}\end{array}$} & $\sum 6$ Pliegues & 48,1 & $+.10,3$ & 51,7 & $+.17,1$ & 57,2 & $+.17,6$ & 50,9 & $+.18,3$ & ${ }^{(* *)} 45,7$ & $+.16,0$ & 52,1 & $+.14,0$ \\
\hline & KG Piel & 3,7 & $+.0,2$ & 3,7 & $+.0,3$ & 3,7 & $+.0,3$ & 3,7 & $+.0,3$ & 3,7 & $+.0,3$ & 3,5 & $+.0,3$ \\
\hline & KG Grasa & 14,6 & $+.2,0$ & 15,2 & $+.2,6$ & 15,8 & $+.3,5$ & 15,0 & $+.2,9$ & 15,1 & $+.4,1$ & 14,2 & $+.2,6$ \\
\hline & KG Muscular & 33,0 & $+.4,2$ & 29,4 & $+.3,3$ & 31,7 & $+.4,7$ & 31,6 & $+.3,5$ & 30,4 & $+.6,2$ & 31,0 & $+.3,4$ \\
\hline & KG Residual & 8,1 & $+.0,8$ & 7,6 & $+.0,9$ & 8,0 & $+.0,8$ & 8,5 & $+.1,1$ & 7,9 & $+.0,9$ & 7,3 & $+.0,8$ \\
\hline & KG Osea & 8,2 & $+.1,0$ & 7,6 & $+.1,0$ & 8,2 & $+.0,9$ & 7,7 & $+.1,4$ & 7,8 & $+.1,1$ & 7,8 & $+.0,7$ \\
\hline & Kg MMES & 5,7 & $+.0,8$ & 5,1 & $+.0,7$ & 5,3 & $+.0,9$ & 5,3 & $+.0,8$ & 4,9 & $+.0,8$ & 5,1 & $+.0,6$ \\
\hline & Kg MMEI & 15,9 & $+.1,6$ & ${ }^{(*)} 13,8$ & $+.1,6$ & ${ }^{(*)} 13,8$ & $+.1,3$ & ${ }^{(*)} 13,8$ & $+.2,1$ & 15,4 & $+.1,8$ & 15,3 & $1+., 7$ \\
\hline & Endo & 2,2 & $+.0,6$ & 2,2 & $+.1,0$ & 2,6 & $+.0,9$ & 2,1 & $+.0,9$ & 1,9 & $+.0,9$ & 3,0 & $+.1,0$ \\
\hline & Meso & 5,3 & $+.0,9$ & 4,5 & $+.0,8$ & 5,1 & $+.0,9$ & 4,6 & $+.0,9$ & 4,5 & $+.1,3$ & 5,0 & $+.1,0$ \\
\hline & Ecto & 2,4 & $+.0,8$ & 2,8 & $+.0,9$ & 2,3 & $+.0,9$ & 2,5 & $+.0,6$ & 2,8 & $+.1,1$ & 2,4 & $+.0,8$ \\
\hline & $\mathrm{X}$ & 0,2 & $+.1,2$ & 0,6 & $+.1,6$ & $-0,3$ & $+.1,9$ & 0,4 & $+.1,3$ & 0,9 & $+.1,7$ & $-0,7$ & $+.1,5$ \\
\hline & $\mathrm{Y}$ & 6,0 & $+.2,4$ & 3,8 & $+.2,0$ & 4,6 & $+.2,3$ & 4,5 & $+.2,3$ & 4,4 & $+.3,1$ & 4,7 & $+.2,6$ \\
\hline \multirow{13}{*}{$\begin{array}{l}\frac{N}{0} \\
\frac{0}{\vec{n}}\end{array}$} & E6 Pliegues & 50,8 & $+.11,4$ & 46,9 & $+.15,6$ & 54,7 & $+.13,0$ & & I & ${ }^{(* *)} 40,8$ & $+.9,9$ & 48,5 & $+.13,0$ \\
\hline & KG Piel & 3,7 & $+.0,2$ & 3,7 & $+.0,2$ & 3,7 & $+.0,2$ & & I & 3,8 & $+.0,3$ & 3,6 & $+.0,2$ \\
\hline & KG Grasa & 15,5 & $+.2,0$ & 14,1 & $+.2,7$ & 15,8 & $+.2,4$ & & / & 13,6 & $+.2,2$ & 14,1 & $+.2,4$ \\
\hline & KG Muscular & 33,0 & $+.3,7$ & 31,3 & $+.3,2$ & 32,5 & $+.3,9$ & & / & 31,1 & $+.4,5$ & 31,4 & $+.2,7$ \\
\hline & KG Residual & 8,0 & $+.0,9$ & 8,0 & $+.0,7$ & 8,2 & $+.1,6$ & & / & 8,1 & $+.1,4$ & 7,4 & $+.0,7$ \\
\hline & KG Osea & 8,2 & $+.0,8$ & 8,1 & $+.0,9$ & 8,1 & $+.0,9$ & & / & 8,0 & $+.0,9$ & 7,8 & $+.0,8$ \\
\hline & Kg MMES & 5,9 & $+.1,0$ & 5,4 & $+.0,7$ & 5,4 & $+.0,7$ & & I & 4,9 & $+.0,7$ & 5,2 & $+.0,5$ \\
\hline & Kg MMEI & 15,3 & $+.1,7$ & 14,7 & $+.2,1$ & 15,2 & $+.1,5$ & & I & 14,9 & $+.1,5$ & 15,5 & $+.1,8$ \\
\hline & Endo & 2,3 & $+.0,6$ & 2,1 & $+.0,9$ & 2,5 & $+.0,8$ & & I & 1,5 & $+.0,5$ & 2,7 & $+.0,9$ \\
\hline & Meso & 4,8 & $+.0,8$ & 5,0 & $+.0,6$ & 4,8 & $+.0,7$ & & I & 4,5 & $+.1,1$ & 4,9 & $+.0,8$ \\
\hline & Ecto & 2,7 & $+.0,7$ & 2,5 & $+.0,8$ & 2,5 & $+.0,5$ & & I & 2,7 & $+.1,0$ & 2,6 & $+.0,9$ \\
\hline & $X$ & 0,4 & $+.1,0$ & 0,3 & $+.1,5$ & 0,0 & $+.1,3$ & & I & 1,2 & $+.1,3$ & $-0,1$ & $+.1,5$ \\
\hline & Y & 4,4 & $+.2,1$ & 5,4 & $+.1,8$ & 4,6 & $+.2,0$ & & I & 4,8 & $+.2,8$ & 4,5 & $+.2,4$ \\
\hline
\end{tabular}


goría sub 16, Ecto-mesomorficos son los equipos de ColoColo, U. de Chile, U. Española y O’Higgins, donde la forma corporal está determinada por el musculo relativo y secundariamente por la linearilidad relativa. Los equipos de Palestino y Everton, presentan una clasificación de Endomesomórficos, donde predomina la forma corporal de acuerdo al musculo relativo y secundariamente la grasa relativa. En la categoría sub 17, se clasifican en Ecto-mesomórficos, las categorías de Colo-Colo, U. de Chile y O’higgins, Ecto- endo-mesomórficos el equipo de Palestino y Everton como Endo-mesomorfico.

En la Figura 1, se aprecia la distribución de ambas categorías en la somatocarta, donde en la categoría sub 16 hay una mayor dispersión de los sujetos y en la categoría sub 17, la dispersión en mucho menor. Se aprecia además la predominancia del mesomorfismo en la forma corporal, al estar los puntos dispuestos hacia la zona superior de la somatocarta.

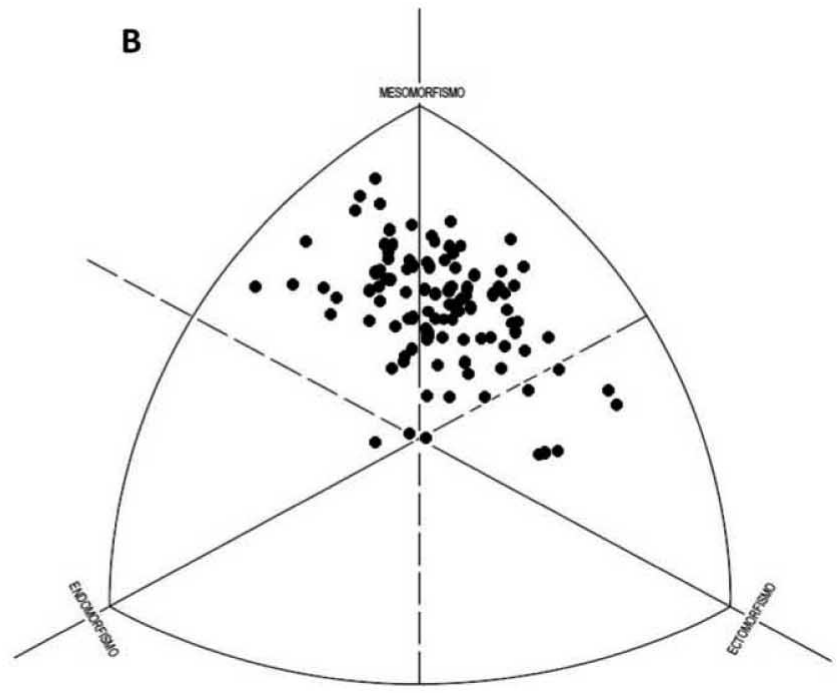

Fig. 1. A. Somatocarta de distribución de los seis equipos sub 16 evaluados. B. Somatocarta de los cinco equipos sub 17 evaluados.

\section{DISCUSIÓN}

Los jóvenes futbolistas, presentan diferencias en la estructura corporal con respecto a futbolistas profesionales, demostrando una falta de desarrollo madurativo y deportivo, que según la evidencia, los hace menos aptos físicamente, para enfrentar exigencias físicas, típicas del futbol profesional.

Una de éstas diferencias es la masa muscular, donde los jugadores profesionales tienen entre 6,8 y $11,2 \mathrm{~kg}$ más de masa muscular total que las categorías sub 16 y entre 5,6 y $10 \mathrm{~kg}$ más de masa muscular total que las categorías sub 17. Esto ocurre al comparar los resultados con los equipos de U de Chile y Everton profesional, medidos por nuestro equipo de trabajo. Estas diferencias se confirman al comparar el peso total de los sujetos, donde las diferencias son de más de $10 \mathrm{~kg}$ (Tabla III).

En nuestro país, partir de los 16 y 17 años, los jóvenes comienzan a ser considerados en el equipo de plantel de honor profesional, agregándolos a las nóminas para los parti-

Tabla III. Comparación de la composición corporal de los equipos de U de Chile profesional, Everton profesional y los equipos sub 16 y sub 17.

\begin{tabular}{lccccc}
\hline Equipos & Peso & Talla & Kg grasa & Kg músculo & 6 pliegues \\
\hline Juveniles sub 16 & 65,3 & 171,4 & 14,8 & 31,0 & 49,1 \\
Juveniles sub 17 & 67,0 & 172,8 & 14,8 & 32,2 & 48,6 \\
U. de Chile profesional & 75,6 & 177,0 & 16,5 & 37,8 & 53,4 \\
Everton profesional & 77,1 & 178,0 & 14,2 & 42,2 & 39,3 \\
\hline
\end{tabular}


dos. Según las comparaciones de la Tabla III, la menor cantidad de masa muscular, podría disminuir el rendimiento físico o estar en desventaja física frente a un jugador más alto y con mayor masa muscular, especialmente en el juego 1 contra 1 .

Los kg de masa grasa no presentan tantas diferencias con los equipos profesionales, como en otros estudios se confirma (Tsolakis \& Vagenas, 2010) comprobando que la diferencia en el peso, es fundamentalmente de masa muscular. La masa grasa es característica de los sujetos que poseen un buen rendimiento físico, especialmente aeróbico. Considerando que los estudios en rendimiento físico en el futbol, muestran que los jugadores profesionales tienen un consumo máximo de oxígeno (VO2 max) entre 56,8 y 67,6 $\mathrm{mL} \cdot \mathrm{kg}-1 \cdot \min$ (Alhazzaa et al., 2001; Bangsbo \& Lindquist, 1992; Bangsbo et al., 1991; Casajus, 2001; Davis et al., 1992; Rhodes et al., 1986; Strudwick et al., 2002; Wisloff, 1998) y recorren entre 10 y $11 \mathrm{~km}$ por partido (Barros et al., 2007; Bangsbo \& Michalsik, 2002; Tumilty, 1993).

La menor masa muscular de estos sujetos, podría además aumentar las posibilidades de sufrir lesiones deportivas musculares o articulares, al incluir a estos jóvenes deportistas, aún no desarrollados, al futbol profesional.
Sobre todo cuando emigran a otros clubes deportivos, como lo demuestra un estudio Finlandés en futbol europeo (Arnason et al.), donde las lesiones de los deportivas ocurren por cambios en la posición de juego entre u equipo y otro.

La masa muscular de los sujetos es menor que la de futbolistas profesionales, por lo que no están aptos para ser incluidos dentro del equipo de honor en los partidos de competencia, con el riesgo de sufrir lesiones y de tener un rendimiento físico menor.

\section{AGRADECIMIENTOS}

Al cuerpo técnico y a los jóvenes jugadores voluntarios de los equipos de Colo Colo, Universidad de Chile, Palestino, Unión Española, O’Higgins de Rancagua y Everton de Viña del Mar. A la Escuela de Nutrición y Dietética de la Facultad de medicina de la Universidad Mayor. A la Dirección de investigación e Innovación de la Pontificia Universidad Católica de Valparaíso, Valparaiso, Chile, por su constante apoyo.

JORQUERA, A. C.; RODRÍGUEZ, R. F.; TORREALBA, V. M. I. \& BARRAZA, G. F. Body composition and somatotype of chilean soccer players sub 16 y sub 17. Int. J. Morphol., 30(1):247-252, 2012.

SUMMARY: Youth soccer is becoming increasingly professionalized to the point of being admitted to adult teams playing in the top leagues. For this to happen it is necessary that young athletes are physically fit to meet the physical demands of professional soccer. Anthropometric assessment was performed in a total of 217 subjects divided into two groups, sub 16 and sub 17. The young players have differences in body structure with respect to professional players, showing a lack of sports development and maturation, which the evidence, the less physically fit to face the physical demands are typical of professional soccer. The muscle mass of subjects is less than professional players, so they are not suitable for inclusion in the professional team in competition matches, with the risk of injury and having a lower physical performance.

\section{KEY WORDS: Anthropometry; Body composition; Muscle mass; Soccer.}

\section{REFERENCIAS BIBLIOGRÁFICAS}

Al-Hazzaa, H. M.; Almuzaini, K. S.; Al-Refaee, S. A.; Sulaiman, M. A.; Dafterdar, M. Y.; Al-Ghamedi, A. \& Al-Khuraiji, K. N. et al. Aerobic and anaerobic power characteristics of Saudi elite soccer players. J. Sports Med. Phys. Fitness, 41:54-61, 2001.

Arnason, A.; Sigurdsson, S. B.; Gudmundsson, A.; Holmei, I.; Engebretsen, L. \& Bahr, R. Physical Fitness, Injuries, And Team Performance In Soccer. Med. Sci. Sports Exerc., 36:27885, 2004.

Bangsbo, J. \& Lindquist, F. Comparison of various exercise tests with endurance performance during soccer in professional players. Int. J. Sports Med., 13:125-32, 1992.
Bangsbo, J.; Norregaard, L. \& Thorso, F. Activity profile of competition soccer. Can. J. Sport Sci., 16:110-6, 1991.

Barros, R.; Misuta, M.; Menezes, R.; Figueroa, P.; Moura, F.; Cunha, S.; Anido, R. \& Leite, N. Analysis of the distances covered by first division Brazilian soccer players obtained with an automatic tracking method. J. Sports Sci. Med., 6:233-42, 2007.

Bangsbo, J. \& Michalsik, L. Assessment of the physiological capacity of elite soccer players. In: Science and Football IV. Spinks, W.; Reilly, T. \& Murphy, A. (Eds.). London, Routledge, 2002. pp.53-62. 
Carter, J. E. L. The Heath-Carter Somatotype method. San Diego, San Diego State University Syllabus Service, 2002.

Carter, J. E. L. \& Heath, B. H. Somatotyping - Development and Applications. Cambridge, Cambridge University Press, 1990.

Casajus, J. A. Seasonal variation in fitness variables in professional soccer players. J. Sports Med. Phys. Fitness, 41:463-9, 2001.

Davis, J. A.; Brewer, J. \& Atkin, D. Pre-season physiological characteristics of English first and second division soccer players. J. Sports Sci., 10:541-7, 1992.

Drinkwater, D. T. An anatomically deroved method for the anthropometric estimation of human body composition. Ph.D. Thesis, Simon Fraser University, 1984.

Kerr, D. A. An anthropometric method for the fracitionation of skin, adipose, muscle, bone and residual tissue masses in males and females age 6 to 77 years. M. Sc. Thesis. Simon Fraser University, 1988.

Krustrup, P. \& Bangsbo, J. Physiological demands of top-class soccer refereeing in relation to physical capacity: Effect of intense intermittent exercise training. J. Sport Sci., 19:881-91, 2001.

Krustrup, P.; Mohr, M.; Amstrup, T.; Rysgaard, T.; Johansen, J.; Steensberg, A.; Pedersen, P. K. \& Bangsbo, J. The Yo-Yo Intermittent Recovery Test: Physiological response, reliability, and validity. Med. Sci. Sport Exerc., 35:697-705, 2003.

Krustrup, P.; Mohr, M.; Ellingsgaard, H. \& Bangsbo, J. Physical demands during an elite female soccer game: Importance of training status. Med. Sci. Sports Exerc., 37:1242-8, 2005.

Malina, R. M.; Eisenmann, J. C.; Cumming, S. P.; Ribeiro, B. \& Aroso, J. Maturity-associated variation in the growth and functional capacities of youth football (soccer) players 13-15 years. Eur. J. Appl. Physiol., 91:555-62, 2004.

Mohr, M.; Krustrup, P. \& Bangsbo, J. Match performance of highstandard soccer players with special reference to development of fatigue. J. Sports Sci., 21:519-28, 2003.

Mohr, M.; Krustrup, P. \& Bangsbo, J. Fatigue in soccer: A brief review. J. Sport Sci., 23:593-9, 2005.

Norton, K. \& Olds, T. Antropometrica. Marrickville, Ed. Southwood Press, 1996.

Rhodes, E. C.; Mosher, R. E.; Mckenzie, D. C.; Franks, I. M.; Potts, J. E. \& Wenger, H. A.. Physiological profiles of the Canadian Olympic Soccer Team. Can. J. Appl. Sport Sci., 11:31-6, 1986.

Rodriguez, R. F. J.; Almagià, F. A. A. \& Berral, R. F. J. Estimación de la masa muscular de los miembros apendiculares, apartir de densitometría fotónica dual (DEXA). Int. J. Morphol., 28:1205-10, 2010.
Strudwick, A.; Reilly, T. \& Doran, D. Anthropometric and fitness profiles of elite players in two football codes. J. Sports Med. Phys. Fitness, 42:239-42, 2002.

Tumilty, D. Physiological characteristics of elite soccer players. Sports Med., 16:80 -96, 1993

Tsolakis, C. \& Vagenas, G. Anthropometric, Physiological and Performance Characteristics of Elite and Sub-elite Fencers. $J$. Hum. Kinet., 23:89-95, 2010.

Wisloff, U.; Helgerud, J. \& Hoff, J. Strength and endurance of elite soccer players. Med. Sci. Sports Exerc., 30:462-7, 1998.

Dirección para correspondencia:

Fernando Rodríguez Rodríguez

Avda. El Bosque 1290 Casilla 4059

Pontificia Universidad Católica de Valparaíso

Valparaíso

CHILE

Email: fernando.rodriguez@ucv.cl

Recibido : 22-11-2011

Aceptado: 19-12-2011 